\title{
Critical limb ischaemia due to thrombosis of the right subclavian artery; a rare presentation of nephrotic syndrome
}

\author{
Jayasekara JMS, Palangasinghe DR, Hewavithana JS, Dahanayake NJ \\ Teaching Hospital, Karapitiya, Galle, Sri Lanka.
}

Correspondance: Dr. Swarnamali Jayasekara

e-mail: swarnamalij2005@gmail.com

https://orcid.org/0000-0002-6378-2463

\section{Introduction}

Thromboembolism is a well-known complication of nephrotic syndrome (NS) (1). The incidences of both venous and arterial thrombosis are higher in patients with NS compared to general population (1). Venous thrombotic complications have frequently been observed (2). Arterial complications are rare and may lead to serious and life-threatening complications depending on the vessels involved and associated with poor prognosis (2-5).

Hypercoaguability in NS is multifactorial; has been attributed to alterations in blood levels of factors involved in the coagulation and fibrinolytic systems, alterations in platelet function, venous stasis, haemoconcentration, increased blood viscosity and possibly the administration of steroids (6).

The deep veins in the legs, the inferior vena cava, the superior vena cava, and the renal veins are particularly involved among veins and there are case reports of cerebral venous sinus thrombosis $(1,2)$. Though rare, involved arterial sites include aortic, renal, femoral, mesenteric, coronary, and cerebral arteries. The commonest site of arterial thrombosis is the femoral artery, occurring mainly in children with NS (5).

Here we report a case of critical limb ischaemia due to thrombosis of the right subclavian artery in an adult as a rare presentation of nephrotic syndrome.

\section{Case Report}

A 29 year-old patient with bronchial asthma and primary hypothyroidism presented with generalized oedema for one month, intermittent claudication of right upper limb for three weeks followed by pain at rest for two days. She was treated with diuretics for one month. There was reduction in urine output one day prior to admission. She was on oral contraceptive pills (OCP) for two years.

On examination there was generalized oedema with bilateral moderate pleural effusions and ascites. Right upper limb was cold with no radial, ulnar or brachial pulses with saturation of $68 \%$ on air (saturation of the left upper limb was $96 \%$ on air). Her pulse rate was $104 \mathrm{bpm}$ and blood pressure was $120 / 70 \mathrm{mmHg}$. Rest of the examination was unremarkable.

Evaluation revealed nephrotic range proteinuria (6g/day) with bland urinary sediments, hypoalbuminaemia $(15 \mathrm{~g} / \mathrm{L})$, hypercholesterolaemia (398 $\mathrm{mg} / \mathrm{dl}$ ) with evidence of acute kidney injury (blood urea (BU) - $171 \mathrm{mg} / \mathrm{dL}$, serum creatinine (S. Cr.) $206 \mu \mathrm{mol} / \mathrm{L}$ ) and dehydration ( $\mathrm{Hb} 17.1 \mathrm{~g} / \mathrm{dL}$, haematocrit $57 \%$ with normal other cell lines) which improved with hydration (BU - $92 \mathrm{mg} / \mathrm{dl}$, S.Cr - 116 $\mu \mathrm{mol} / \mathrm{L}, \mathrm{Hb} 11.3 \mathrm{~g} / \mathrm{dL}$, haematocrit 44\%). Upper limb venous doppler revealed thrombosis of bilateral axillary veins with extension into brachial vein on right side. CT angiogram showed aberrant right subclavian artery with thrombosis of right subclavian, axillary and brachial arteries. Her autoimmune and thrombophilia screenings were negative.

She responded well to conventional heparin followed by subcutaneous enoxaparin and warfarin, high dose steroids, intravenous frusemide and intravenous albumin. 

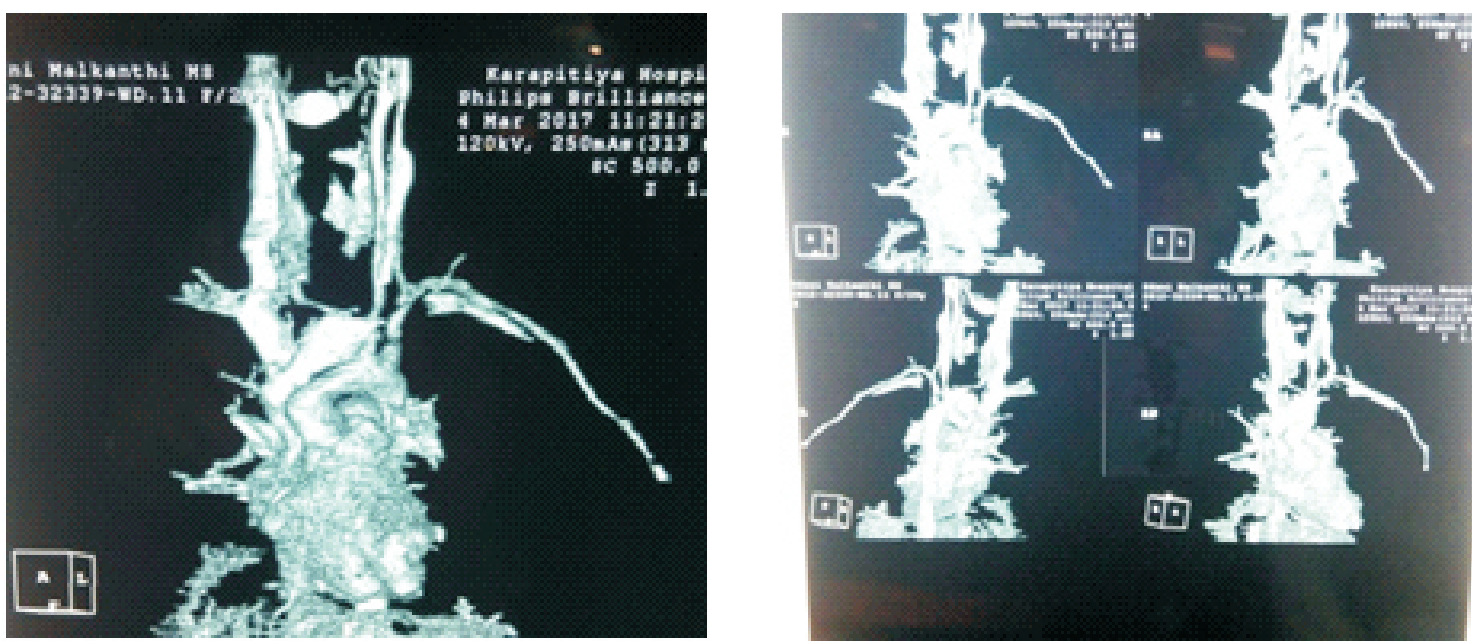

Figure 1: CT angiogram showing abrupt cut off right subclavian artery with thrombosis

\section{Discussion}

This 29 year-old female presented with intermittent claudication of the right upper limb due to thrombosis of the aberrant right sided subclavian artery. At the same time she was diagnosed to have NS. In addition she was on OCP for 2 years and was dehydrated on admission. Thrombosis in general and arterial thrombosis in particular is a significant and potentially serious problem in patients with NS $(5,8)$. Most cases of venous thromboembolism associated with NS reported in the literature have a preceding diagnosis of NS (5).

The increased propensity of thromboembolism in nephrotic patients is postulated to be a result of increased excretion of antithrombotic factors (antithrombin III, plasminogen, protein C, and protein $\mathrm{S}$ ) by the affected kidneys and increased production of pro-thrombotic factors like fibrinogen by the liver (5).

Interestingly our patient had aberrant right side subclavian artery on which the origin is narrower than the usual one. She was on OCP and it is a well-known predisposing factor for thrombosis. Also she was dehydrated on admission without marked peripheral oedema with haemoconcentration probably due to pretreatment with diuretics. Hypercoaguable state of NS together with the effect of OCP, aberrancy of right subclavian artery and dehydration altogether contributed to thrombosis in our patient.
Arterial thrombosis can be diagnosed with the use of duplex scanning, CT angiography, or magnetic resonance angiography (6).

Once the diagnosis is established, anticoagulation therapy should be started. Patient can be treated with conventional or low molecular weight heparin, followed by oral warfarin and antiplatelet agents. Thrombo-embolectomy or thrombolytic therapy is indicated in patients with ischemic limbs due to arterial thromboembolism. Attempts to reduce the degree of proteinuria, hyperlipidaemia, and hypertension should be made in all patients with NS (6).

The patient was treated with conventional heparin followed by subcutaneous enoxaparin and warfarin and remarkable improvement was noted.

To conclude, arterial thrombosis is a rare but a serious complication of NS at first presentation which needs high index of clinical suspicion for prompt diagnosis and immediate treatment is needed to prevent further complications.

\section{Conclusions}

Although rare both arterial and venous thrombosis can occur with NS. Hypercoaguable state of NS together with the effect of OCP, right subclavian artery aberrancy and dehydration, all have contributed to thrombosis in this patient. We were able to save the limb due to prompt diagnosis and early treatment. 


\section{References}

1. Uptodate 21.6. Renal vein thrombosis and hypercoagulable state in nephrotic syndrome, Acute arterial occlusion of the lower extremities (acute limb ischaemia).

2. Ibrahim H. Fahal, Peter McClelland, Charles R.M. Hay' and Gordon M. Bell. Arterial thrombosis in the nephrotic syndrome; Postgrad Med J, (1994) 70: 905 - 9. [Google Scholar].

3. Kyoung Hee Han, Ji Youn Park, Seung-Kee Min, Il-Soo Ha, Hae Il Cheong, and Hee Gyung Kang. Bilateral iliac and popliteal arterial thrombosis in a child with focal segmental glomerulosclerosis. Korean Journal of pediatrics , 2016 May; 59(5): 242-5. [PubMed].

4. Niranjan Tachamo, Rashmi Dhital, Bidhya Timilsina, Salik Nazir, Saroj Lohani, Dilli Ram Poudel. Popliteal arterial thrombosis in nephrotic syndrome. Journal of Community Hospital Internal Medicine Perspectives; 31 Mar 2017 [PubMed].
5. Hasan F Al-Azzawi, Onyekachi C Obi, Javeryah Safi, Mingchen Song. Nephrotic syndrome - induced thromboembolism in adults. 2016 Apr-Jun; 6(2): 85-8 [PubMed].

6. BK Brahmbhatt, A Mathew, R Rajesh, G Kurian, VN Unni; Brachiocephalic artery thrombosis in adult nephrotic syndrome. Indian Journal of Nephrology, July-September, 2011,21(3): 204-7. [Google Scholar].

7. Kularatne SAM, Dewapura DS. Acute pulmonary embolism in a patient with nephrotic syndrome. Ceylon Medical Journal, 1993; 38(2)-90. 